Journal of Mathematical Physics, Analysis, Geometry

2015, vol. 11, No. 3, pp. 207-235

\title{
On Stability and Stabilization of Perturbed Time Scale Systems with Gronwall Inequalities
}

\author{
B. Ben Nasser ${ }^{1}$, K. Boukerrioua ${ }^{2}$, and M.A. Hammami ${ }^{1}$ \\ ${ }^{1}$ University of Sfax, Department of Mathematics, Faculty of Sciences of Sfax \\ B.P 1171, Sfax, 3000, Tunisia \\ E-mail: bacem.bennasser@yahoo.fr \\ MohamedAli.Hammami@fss.rnu.tn \\ ${ }^{2}$ University 08 mai 1945 of Guelma, Department of Mathematics, Avenue 19 May 1956 \\ B.P 401, Guelma, Algeria \\ E-mail: khaledv2004@yahoo.fr
}

Received January 2, 2014, revised January 27, 2015

In the paper, new nonlinear time scale integral inequalities are given. By means of the explicit integral bounds, we derive sufficient conditions for the uniform asymptotic stability of perturbed systems on time scales. In the sequel, basing on Lyapunov's direct method, we develop the required type of stability.

Key words: time scales, perturbed systems, Gronwall inequalities, quadratic stability.

Mathematics Subject Classification 2010: 34D05, 34D10, 34D23, 37C75, 93D20.

\section{Introduction}

The time scale theory, which has lately attracted much attention of many authors $[6,7]$, stems from doctoral dissertation research performed by Stefan Hilger in 1988 in [16] and continued in [17]. The field of dynamic equations on time scale gathers bonds and expands the standard theories of differential and difference equations. Such an extension gives us an opportunity to regard the continuous and discrete cases simultaneously. The stability theory is one of the important branches of differential equation theory. Multiple research activities are mainly focused on this approach (see [10] and [22]). Some of the results are obtained for time scale dynamic equations in [21]. In particular, the time scale stability analysis was launched in 1990s with the work of Aulbach and Hilger [2]. A particular attention was paid to the linear delta differential equations and to the

(c) B. Ben Nasser, K. Boukerrioua, and M.A. Hammami, 2015 
asymptotic stability using Lyapunov approach in [18]. Latterly, there have been several papers (Dacunha [13], Peterson and Raffoul [31], Bohner and Martynyuk [8], Martynyuk [27], Martynyuk et al. [28]) studying the properties of exponential stability of dynamical time scale systems. Perturbation theory is a pertinent discipline for the applications of time scale dynamics which is a compilation of methods systematically used to evaluate the global behavior of solutions to both difference and differential equations. Within the same context, we are concerned about developing some categories of perturbed time scale dynamics having the formula

$$
x^{\Delta}(t)=A(t) x+F(t, x),
$$

where $A: \mathbb{T} \rightarrow \mathbb{R}^{n * n}$ is a regressive matrix valued function and an rd-continuous vector valued function $F: \mathbb{T} \times \mathbb{R}^{n} \rightarrow \mathbb{R}^{n}$ (see the definition of the right-dense continuous function 2.5), satisfying $\|F(t, 0)\| \leq F_{0}$ on $\mathbb{T}$, regarded as a disturbance to the linear system

$$
x^{\Delta}(t)=A(t) x .
$$

Concerning the continuous type, i.e., $\mathbb{T}=\mathbb{R}$, several results related to asymptotic stability using Lyapunov techniques were obtained (see $[5,14,15]$ ). At first, one of the methods of the perturbation theory was referred to integral inequalities to quest some type of stability. In the last few years, the search was directed to the time scale integral inequalities by using diverse techniques and some significant results were obtained (see $[8,30,24,25])$. We are interested in further generalizing some nonlinear dynamic integral estimations. We show generalizations of some dynamic inequalities for handling the qualitative behavior of trajectories of time scale perturbed systems. Here we get the explicit bounds of solutions through certain adequate time scale integral inequalities. Lyapunov's direct method is one of the tools used for investigating the stability of a certain class of systems, namely, a perturbed one. The idea of this method is to develop the stability of the given system by measuring the rate change of the energy of the modulated system. Lyapunov's stability was studied for time scale dynamics by Kaymakcalan [20]. Hoffacker and Tisdell [19] also studied Lyapunov's direct method. Specifically, Dacunha [13] extended Lyapunov's second (direct) method for application to the stability analysis of certain classes of dynamics (e.g., slowly time-varying systems), developed and solved a time scale algebraic Lyapunov equation. In this work, we obtain some stability results for the considered system in the spirit of Lyapunov's direct method even for the case where $V^{\Delta}$ is nonnegative semi-definite. Basing on the work of Dacunha [13], we expect that under certain conditions imposed on their perturbations and some matrix inequalities, involving a symmetric definite positive matrix function, some stability properties are insured for solutions of system (2). As an application, we give a result of stabilizability of the control nonlinear time scale system. The paper starts with an 
appropriate framework of time scale calculus. We also introduce some definitions of stability needed to characterize asymptotic trajectory behaviors for or near the origin. Establishing some time scale integral inequalities improves studying of stability of perturbed dynamics, which is one of the main points of our results. Finally, the stability asymptotic convergence will be ensured by the time scale Lyapunov's direct method.

\section{Time Scale and Stability Analysis}

\subsection{Time scale essentials}

First, we will briefly mention some basic definitions and results of time scale calculus for reader's convenience, as they are detailed in the books of M. Bohner and A. Peterson (see [7] and [6]).

Definition 2.1. A time scale $\mathbb{T}$ is a nonempty closed subset of the set $\mathbb{R}$ of real numbers.

The standard cases comprise $\mathbb{T}=\mathbb{R}, \mathbb{T}=\mathbb{Z}, \mathbb{T}=h \mathbb{Z}$ for $h>0$. We assume throughout that $\mathbb{T}$ has the topology that is inherited from the standard topology on $\mathbb{R}$. The notation $\mathbb{T}_{a}^{+}$, and so on, will denote time scales intervals such that $\mathbb{T}_{a}^{+}=\{t \in \mathbb{T} ; a \leq t\}$, where $a \in \mathbb{T}$. Since a time scale may not be connected, we need the following concept of jump operators.

Definition 2.2. For $t \in \mathbb{T}$, we define:

- the forward jump operator $\sigma: \mathbb{T} \rightarrow \mathbb{T}$ by $\sigma(t):=\inf \{s \in \mathbb{T} ; s>t\}$;

- the backward jump operator $\rho: \mathbb{T} \rightarrow \mathbb{T}$ by $\rho(t):=\sup \{s \in \mathbb{T} ; s<t\}$;

- the graininess function $\mu: \mathbb{T} \rightarrow \mathbb{R}_{+}$by $\mu(t):=\sigma(t)-t$.

Finally, we define the set $\mathbb{T}^{\kappa}$ in the following way: $\left.\mathbb{T}^{\kappa}:=\mathbb{T}-\right] \rho(\sup \mathbb{T})$, sup $\left.\mathbb{T}\right]$ if $\sup \mathbb{T}<\infty$, otherwise, $\mathbb{T}^{\kappa}:=\mathbb{T}$.

The jump operators $\sigma$ and $\rho$ allow the classification of points in $\mathbb{T}$ in the following way:

Definition 2.3. A non-maximal element $t \in \mathbb{T}$ is said to be right-dense if $\sigma(t)=t$, right-scattered if $\sigma(t)>t$, left-dense if $\rho(t)=t$, left-scattered if $\rho(t)<t$.

In the case $\mathbb{T}=\mathbb{R}$, we have $\sigma(t)=t$, and if $\mathbb{T}=h \mathbb{Z}$ for $h>0$, then $\sigma(t)=t+h$. 
Definition 2.4. Let $f$ be a function defined on $\mathbb{T}$ and let $t \in \mathbb{T}^{\kappa}$. Then we define $f^{\Delta}(t)$ to be the number (provided it exists) with the following property: for any $\varepsilon>0$, there is a neighborhood $U$ of $t$ (i.e., $U=] t-\delta, t+\delta[\mathbb{T}$ for some $\delta$ ) such that

$$
\left|[f(\sigma(t))-f(s)]-f^{\Delta}(t)[\sigma(t)-s]\right| \leq \varepsilon|\sigma(t)-s|
$$

for all $s \in U$. We call $f^{\Delta}(t)$ the delta derivative of $f$ at $t$. Moreover, we say that $f$ is delta differentiable on $\mathbb{T}^{\kappa}$ provided $f^{\Delta}(t)$ exists for all $t \in \mathbb{T}^{\kappa}$.

$\mathrm{R}$ e $\mathrm{m}$ a $\mathrm{rk}$ 2.1. Note that in the case $\mathbb{T}=\mathbb{R}$, we have $f^{\Delta}(t)=f^{\prime}(t)$ and when $\mathbb{T}=\mathbb{Z}$, we get $f^{\Delta}(t)=\Delta f(t)=f(t+1)-f(t)$.

\section{Definition 2.5.}

- A function $f: \mathbb{T} \rightarrow \mathbb{R}$ is called rd-continuous (denote $f \in \mathcal{C}_{r d}:=\mathcal{C}_{r d}(\mathbb{T}, \mathbb{R})$ ), if

(i) $f$ is continuous at every right-dense point on $\mathbb{T}$,

(ii) $\lim _{s \rightarrow t^{-}} f(s)$ exists and it is finite at every left-dense point $t \in \mathbb{T}$.

- A function $f: \mathbb{T} \times \mathbb{R}^{n} \rightarrow \mathbb{R}^{n}$ is said to be rd-continuous, if $h$ defined by $h(t)=f(t, x(t))$ is rd-continuous for any continuous function $x: \mathbb{T} \rightarrow \mathbb{R}^{n}$.

Definition 2.6. Let $f \in \mathcal{C}_{r d}(\mathbb{T}, \mathbb{R})$. Then a function $F: \mathbb{T} \rightarrow \mathbb{R}$ is called antiderivative of $f$ on $\mathbb{T}$ if it is differentiable on $\mathbb{T}^{\kappa}$ and satisfies $F^{\Delta}(t)=f(t)$ for any $t \in \mathbb{T}^{\kappa}$. In this case, we define the Cauchy integral

$$
\int_{a}^{t} f(s) \Delta s=F(t)-F(a) \text { for all } a, t \in \mathbb{T} .
$$

For a more general definition of the delta integral, see [6, section 1.4].

\section{Definition 2.7.}

- An rd-continuous function $p: \mathbb{T} \rightarrow \mathbb{R}$ is said to be regressive (denote $p \in \mathcal{R}(\mathbb{T}, \mathbb{R})$ ) if, at all $t \in \mathbb{T}^{\kappa}$,

$$
1+\mu(t) p(t) \neq 0
$$

- $\mathcal{R}^{+}$is the set of all positively regressive elements of $\mathcal{R}$ such that

$$
\mathcal{R}^{+}=\{p \in \mathcal{R}: 1+\mu(t) p(t)>0 \text { for all } t \in \mathbb{T}\} .
$$

Throughout we let $t_{0} \in \mathbb{T}$. 
Definition 2.8. Let $p \in \mathcal{R}$. Consider the regressive dynamic initial valueproblem

$$
x^{\Delta}(t)=p(t) x, x\left(t_{0}\right)=1 .
$$

The exponential function $x(t)=e_{p}\left(t, t_{0}\right)$ is the unique solution of (3) on $\mathbb{T}$.

For what follows, we need several theorems presented below.

Theorem 2.1. (Comparison Theorem). Let $x, f \in \mathcal{C}_{r d}$ and $p \in \mathcal{R}^{+}$. Then

$$
x^{\Delta}(t) \leq p(t) x(t)+f(t), \text { for all } t \in \mathbb{T},
$$

implies

$$
x(t) \leq x\left(t_{0}\right) e_{p}\left(t, t_{0}\right)+\int_{t_{0}}^{t} e_{p}(t, \sigma(\tau)) f(\tau) \Delta \tau \text { for all } t \in \mathbb{T} .
$$

Theorem 2.2. (Gronwall's Inequality). Let $x, f \in \mathcal{C}_{r d}$ and $p \in \mathcal{R}^{+}\left(\mathbb{T}, \mathbb{R}_{+}\right)$. Then

$$
x(t) \leq f(t)+\int_{t_{0}}^{t} x(\tau) p(\tau) \Delta \tau, \text { for all } t \in \mathbb{T},
$$

implies

$$
x(t) \leq f(t)+\int_{t_{0}}^{t} e_{p}(t, \sigma(\tau)) f(\tau) p(\tau) \Delta \tau \text { for all } t \in \mathbb{T} .
$$

Now, we find the comparison relations from [9] between the general exponential function on time scales and the classical exponential function.

Lemma 2.1. For a nonnegative $p$ with $-p \in \mathcal{R}^{+}$, we have the inequalities

$$
1-\int_{t_{0}}^{t} p(u) \Delta u \leq e_{-p}\left(t, t_{0}\right) \leq \exp \left\{-\int_{t_{0}}^{t} p(u) \Delta u\right\} \text { for all } t \in \mathbb{T}_{t_{0}}^{+} .
$$

If $p$ is rd-continuous and nonnegative, then

$$
1+\int_{t_{0}}^{t} p(u) \Delta u \leq e_{p}\left(t, t_{0}\right) \leq \exp \left\{\int_{t_{0}}^{t} p(u) \Delta u\right\} \text { for all } t \in \mathbb{T}_{t_{0}}^{+} .
$$




\subsection{Definitions of stability}

We consider the dynamic system defined on a time scale $\mathbb{T}$ as below

$$
x^{\Delta}(t)=f(t, x),
$$

where $x \in \mathbb{R}^{n}$ and $f: \mathbb{T} \times \mathbb{R}^{n} \rightarrow \mathbb{R}^{n}$ is rd-continuous with $\|f(t, 0)\| \leq f_{0}$. Also assume that the conditions for the existence and uniqueness of solutions of this system are satisfied for all initial states $\left(t_{0}, x_{0}\right) \in \mathbb{T} \times \mathbb{R}^{n}$. For further explanations concerning the existence and uniqueness of solutions of nonlinear systems, it is recommended to refer to [6, section 8.2]. Designate any solution of (6) with the initial state $\left(t_{0}, x_{0}\right)$ by $x(t)=x\left(t, t_{0}, x_{0}\right)$.

Definitions of stability of time scale systems are obtained by a slight modification of their standard definitions for ordinary differential equations. At first, we start from the stability characterization of trajectories closely related to the stability of the trivial null solution of the corresponding variational equation. So, the origin is required to be an equilibrium point.

\section{Definition 2.9.}

- The zero solution of (6) is said to be uniformly stable if for any $\varepsilon>0$ there exists a $\delta=\delta(\varepsilon)>0$ such that

$$
\left\|x\left(t_{0}\right)\right\|<\delta \Rightarrow\left\|x\left(t, t_{0}, x_{0}\right)\right\|<\varepsilon, \forall t \in \mathbb{T}_{t_{0}}^{+} .
$$

- The zero solution of (6) is said to be uniformly asymptotically stable if it is uniformly stable and there exists $c>0$, independent of $t_{0}$, such that

$$
\left\|x\left(t_{0}\right)\right\|<c \Rightarrow \lim _{t \rightarrow+\infty}\left\|x\left(t, t_{0}, x_{0}\right)\right\|=0
$$

uniformly in $t_{0}$.

- The zero solution of (6) is said to be uniformly globally asymptotically stable if it is uniformly stable, $\delta(\varepsilon)$ can be chosen to satisfy $\lim _{\varepsilon \rightarrow+\infty} \delta(\varepsilon)=+\infty$, and the relation (8) holds for every $x\left(t_{0}\right) \in \mathbb{R}^{n}$.

- The zero solution of (6) is said to be uniformly exponentially stable if there exist the constants $\lambda, \gamma>0$ with $-\lambda \in \mathcal{R}^{+}$such that for any $t_{0}$ and $x\left(t_{0}\right)$, the corresponding solution satisfies

$$
\|x(t)\| \leq \gamma e_{-\lambda}\left(t, t_{0}\right)\left\|x_{0}\right\| \forall t \in \mathbb{T}_{t_{0}}^{+} .
$$

The positif reals $\gamma$ and $\lambda$ are the so-called growth constants. 
- For $t \in \mathbb{T}$, dynamical system (6) is said to be uniformly practically exponentially stable if there exists a positive decreasing rd-continuous function $r(t)$ vanishing at infinity and positive constants $\gamma, \lambda$, with $-\lambda \in \mathcal{R}^{+}(\gamma$ is independent on any initial state $\left.\left(t_{0}, x_{0}\right)\right)$ such that

$$
\|x(t)\| \leq \gamma e_{-\lambda}\left(t, t_{0}\right)\left\|x_{0}\right\|+r(t) \text { for all } t \geq t_{0} .
$$

$\mathrm{R}$ e $\mathrm{m}$ a $\mathrm{r} \mathrm{k} 2.2$. The last type of the stability aspect relates to the case where the size of initial states can at random increase and the corresponding trajectories converge, in a stable way by a decaying time-scale exponential function, to a small neighborhood of the origin. Especially, the definition below merely serves to characterize the problem of asymptotic stability not for the origin, but for a neighborhood of the origin approximated by a small ball of radius $\bar{r}:=\sup \{r(t), t \in \mathbb{T}\}$ centered at the origin.

Definition 2.10. A mapping $A: \mathbb{T} \rightarrow \mathbf{M}_{n}(\mathbb{R})$ is called regressive if for each $t \in \mathbb{T}^{k}$ the $n \times n$ matrix $I_{n}+\mu(t) A(t)$ is invertible, where $I_{n}$ is the identity matrix. The class of all regressive and rd-continuous functions $A$ from $\mathbb{T}$ to $\mathbf{M}_{n}(\mathbb{R})$ is denoted by $\mathcal{C}_{\text {rd }} \mathcal{R}\left(\mathbb{T}, \mathbf{M}_{n}(\mathbb{R})\right)$.

Definition 2.11. Let $t_{0} \in \mathbb{T}$. The unique matrix-valued solution of the IVP,

$$
X^{\Delta}(t)=A(t) X(t), X\left(t_{0}\right)=I_{n},
$$

where $A \in \mathcal{C}_{r d} \mathcal{R}\left(\mathbb{T}, \mathbf{M}_{n}(\mathbb{R})\right)$, is called the matrix exponential function and it is denoted by $\Phi_{A}\left(t, t_{0}\right)$.

$\mathrm{R}$ e $\mathrm{m}$ a $\mathrm{r} \mathrm{k}$ 2.3. For more properties of the matrix exponential function we refer to [6, section 5.1] and to [12] for its explicit form using Peano-Baker series.

S.K. Choi, D.M. Im, and N. Koo in [11] proved that the stability of (2) is equivalent to the boundedness of all its solutions when $A \in \mathcal{C}_{r d} \mathcal{R}\left(\mathbb{T}, \mathbf{M}_{n}(\mathbb{R})\right)$. Also, DaCunha in [13] proved that the uniform stability of (2) is similar to the uniform boundedness of all its trajectories with respect to the initial point $t_{0}$, given $A \in \mathcal{C}_{r d} \mathcal{R}\left(\mathbb{T}, \mathbf{M}_{n}(\mathbb{R})\right)$.

\section{Main Results}

\subsection{Integral dynamic inequalities}

We begin by recalling Pachpatte's inequality. The following lemma is useful in our main results and its proof in [30] uses the time scale version of the inequality obtained by Sansone and Conti [29, p. 86]. By using Comparison Theorem 2.1 and Gronwall's inequality 2.2 , we get it. 
Lemma 3.1. Let $a \in \mathbb{T}, \chi, \varrho, \vartheta \in \mathcal{C}_{r d}\left(\mathbb{T}, \mathbb{R}_{+}\right)$and $\varrho$ be delta-differentiable on $\mathbb{T}$ with $\varrho^{\Delta}(t) \geq 0$. If

$$
\chi(t) \leq \varrho(t)+\int_{a}^{t} \vartheta(s) \chi(s) \Delta s, t \in \mathbb{T}^{\kappa}
$$

then

$$
\chi(t) \leq \varrho(a) e_{\vartheta}(t, a)+\int_{a}^{t} \varrho^{\Delta}(s) e_{\vartheta}(t, \sigma(s)) \Delta s, t \in \mathbb{T}^{\kappa} .
$$

In this section, some Gronwall nonlinear dynamical integral inequalities on time scales are enumerated by putting a nonlinear term in the integral kernel on the right side.

Lemma 3.2. Let $\phi, \varphi, \psi, \alpha, \chi \in \mathcal{C}_{r d}\left(\mathbb{T}, \mathbb{R}_{+}\right)$and $a \in \mathbb{T}$. Then

$$
\phi(t) \leq \varphi(t)+\psi(t) \int_{a}^{t}\{\alpha(s) \phi(s)+\chi(s)\} \Delta s
$$

implies

$$
\phi(t) \leq \varphi(t)+\psi(t) \int_{a}^{t}\{\alpha(s) \varphi(s)+\chi(s)\} \exp \left[\int_{\sigma(s)}^{t} \alpha(\tau) \psi(\tau) \Delta \tau\right] \Delta s
$$

for all $t \in \mathbb{T}_{a}^{+}$.

P r o o f. For $t \in \mathbb{T}_{a}^{+}$, define a function $z(t)$ as

$$
z(t)=\int_{a}^{t}\{\alpha(s) \phi(s)+\chi(s)\} \Delta s
$$

Thus, we have

$$
\phi(t) \leq \varphi(t)+\psi(t) z(t)
$$

and

$$
z^{\Delta}(t)=\alpha(t) \phi(t)+\chi(t) \leq[\alpha(t) \psi(t)] z(t)+[\alpha(t) \varphi(t)+\chi(t)] .
$$

Using Comparison Theorem 2.1 for (14), it follows that

$$
z(t) \leq \int_{a}^{t} e_{\alpha \psi}(t, \sigma(s))[\alpha(s) \varphi(s)+\chi(s)] \Delta s
$$


Indeed, by exponential approximation (5), we get

$$
e_{\alpha \psi}(t, \sigma(s)) \leq \exp \left(\int_{\sigma(s)}^{t} \alpha(\tau) \psi(\tau) \Delta \tau\right)
$$

This, together with relations (16) and (13), gives the required inequality in (12) for all $t \in \mathbb{T}_{a}^{+}$.

For the same considered aim, we postulate the following result involving a Lipschitz-type function in the integral kernel.

Theorem 3.1. Let $a \in \mathbb{T}, \phi, \varphi, \psi \in \mathcal{C}_{r d}\left(\mathbb{T}, \mathbb{R}_{+}\right)$and $P, Q: \mathbb{T} \times \mathbb{R}_{+} \rightarrow \mathbb{R}_{+}$are $r d$-continuous functions satisfying

$$
0 \leq P(t, x)-P(t, y) \leq Q(t, y)(x-y), t \in \mathbb{T}, x \geq y \geq 0 .
$$

Then

$$
\phi(t) \leq \varphi(t)+\psi(t) \int_{a}^{t} P(s, \phi(s)) \Delta s, \quad t \in \mathbb{T}^{\kappa}
$$

implies

$$
\phi(t) \leq \varphi(t)+\psi(t) \int_{a}^{t} P(s, \varphi(s)) \exp \left[\int_{\sigma(s)}^{t} Q(r, \varphi(r)) \psi(r) \Delta r\right] \Delta s, \quad t \in \mathbb{T}^{\kappa} .
$$

P r o o f. Define a function on $\mathbb{T}$ by

$$
y(t):=\int_{a}^{t} P(s, \phi(s)) \Delta s .
$$

Then, a $\Delta$-derivative of $y$ with respect to $t \in \mathbb{T}^{\kappa}$ yields $y^{\Delta}(t)=P(t, \phi(t))$.

Using Lipchitz condition (17), for any $t \in \mathbb{T}^{\kappa}$, we get

$$
\begin{gathered}
y^{\Delta}(t)=P(t, \phi(t)) \leq P(t, \varphi(t)+\psi(t) y(t)), \\
P(t, \varphi(t)+\psi(t) y(t)) \leq P(t, \varphi(t))+Q(t, \varphi(t)) \psi(t) y(t), \\
y^{\Delta}(t) \leq P(t, \varphi(t))+Q(t, \varphi(t)) \psi(t) y(t) .
\end{gathered}
$$

By delta-integrating relation (19) from $a$ to $t \in \mathbb{T}^{\kappa}$, we have

$$
y(t)=\int_{a}^{t} y^{\Delta}(s) \Delta s \leq \int_{a}^{t} P(s, \varphi(s)) \Delta s+\int_{a}^{t} Q(s, \varphi(s)) \psi(s) y(s) \Delta s .
$$


Define two functions on $\mathbb{T}$,

$$
\varrho(t)=\int_{a}^{t} P(s, \varphi(s)) \Delta s \text { and } \vartheta(t)=Q(t, \varphi(t)) \psi(t) .
$$

Then inequality (20) can be written as

$$
y(t) \leq \varrho(t)+\int_{a}^{t} \vartheta(s) y(s) \Delta s .
$$

By Lemma 3.1, relation (22) becomes

$$
y(t) \leq \int_{a}^{t} P(s, \varphi(s)) e_{\vartheta}(t, \sigma(s)) \Delta s .
$$

From Lemma 2.1, it follows that

$$
e_{\vartheta}(t, \sigma(s)) \leq \exp \left\{\int_{\sigma(s)}^{t} Q(r, \varphi(r)) \psi(r) \Delta r\right\} .
$$

By combining relations (21), (23) and (24), we have that

$$
y(t) \leq \int_{a}^{t} P(s, \varphi(s)) \exp \left\{\int_{\sigma(s)}^{t} Q(r, \varphi(r)) \psi(r) \Delta r\right\} \Delta s,
$$

from which we obtain the desired integral inequality (18).

Lemma 3.3. Assume that $a \in \mathbb{T}, \phi, \varphi, \psi \in \mathcal{C}_{r d}\left(\mathbb{T}, \mathbb{R}_{+}\right)$. Let $R(.,):. \mathbb{T} \times \mathbb{R}_{+} \rightarrow$ $\mathbb{R}_{+}$be an rd-continuous function. In addition, the partial derivative with respect to $x$ is a positive rd-continuous function and monotone decreasing for $x \in \mathbb{R}_{+}^{*}$.

Then

$$
\phi(t) \leq \varphi(t)+\psi(t) \int_{a}^{t} R(u, \phi(u)) \Delta u, t \in \mathbb{T}^{\kappa}
$$

implies

$$
\phi(t) \leq \varphi(t)+\psi(t) \int_{a}^{t} R(u, \varphi(u)) \exp \int_{\sigma(u)}^{t} \frac{\partial R}{\partial x}(r, \varphi(r)) \psi(r) \Delta r \Delta u, \quad t \in \mathbb{T}^{\kappa} .
$$


P r o o f. Applying the mean value theorem for the function $R(t,$.$) , then for$ every $x \geq y>0$ and $t \in \mathbb{T}$, there exists $c \in] y, x[$ such that

$$
R(t, x)-R(t, y)=\frac{\partial R}{\partial x}(t, c)(x-y)
$$

and, therefore, from monotony of partial derivative of $R$ with respect to $x$, we get

$$
R(t, x)-R(t, y) \leq \frac{\partial R}{\partial x}(t, y)(x-y)
$$

which implies that $R$ satisfies relation (17) with $Q(.,)=.\frac{\partial R}{\partial x}(.,$.$) .$

The proof of the above lemma follows now by using the arguments similar to those of Theorem 3.1. We omit the details.

Lemma 3.4. Assume that $a \in \mathbb{T}, \varphi, \psi, \phi, \alpha \in \mathcal{C}_{r d}\left(\mathbb{T}, \mathbb{R}_{+}\right)$, and $\beta: \mathbb{R}_{+} \rightarrow \mathbb{R}_{+}$ is a differentiable increasing function on $] 0,+\infty$ [ with continuous decreasing first derivative $\beta^{\prime}$ on $] 0,+\infty[$. If $\phi$ satisfies

$$
\phi(t) \leq \varphi(t)+\psi(t) \int_{a}^{t} \alpha(u) \beta(\phi(u)) \Delta u, \quad t \in \mathbb{T}^{\kappa}
$$

then we have the bound

$$
\phi(t) \leq \varphi(t)+\psi(t) \int_{a}^{t} \alpha(u) \beta(\phi(u)) \exp \int_{\sigma(u)}^{t} \alpha(r) \beta^{\prime}(\varphi(r)) \psi(r) \Delta r \Delta u, \quad t \in \mathbb{T}^{\kappa} .
$$

P r o o f. The proof is essentially identical to that of Lemma 3.3.

Theorem 3.2. Let $a \in \mathbb{T}$. If $\phi, \varphi, \psi, h \in \mathcal{C}_{r d}\left(\mathbb{T}, \mathbb{R}_{+}\right)$and $P, Q: \mathbb{T} \times \mathbb{R}_{+} \rightarrow \mathbb{R}_{+}$, are rd-continuous functions satisfying condition (17), then for every nonnegative rd-continuous positive function $\phi$ satisfying the inequality

$$
\phi(t) \leq \varphi(t)+\psi(t) \int_{a}^{t}[P(s, \phi(s))+h(s)] \Delta s, t \in \mathbb{T}^{\kappa}
$$

implies

$$
\phi(t) \leq \varphi(t)+\psi(t) \int_{a}^{t}\{P(s, \varphi(s))+h(s)\} \times \exp \left[\int_{\sigma(s)}^{t} Q(r, \varphi(r)) \psi(r) \Delta r\right] \Delta s, t \in \mathbb{T}^{\kappa} .
$$


P r o o f. Let us consider the mapping:

$$
\begin{gathered}
y: \mathbb{T} \rightarrow \mathbb{R}_{+} \\
t \rightarrow y(t):=\int_{a}^{t}\{P(s, \phi(s))+h(s)\} \Delta s .
\end{gathered}
$$

The remainder of the proof is similar to that of Theorem 3.1 and we omit it here.

\subsection{Stability via integral inequalities}

Due to the form of the required system (1) that modulates a perturbation of phenomena registered by a time-varying dynamical linear system (2), there is an explicit form of solutions obtained in the same way as those for the discrete and continuous cases given by Alekseev's formula in 1961 (see [1], or some of its generalizations [26]). Thus, we are going to utilize the recording time scale version of the variation of parameters formula, shown in [8, section 3], to analyze stability estimations.

In what follows, we note with $x()=.x\left(., t_{0}, x_{0}\right)$ an arbitrary solution of the perturbed time scale system (1).

Theorem 3.3. Consider the regressive time-varying perturbed system

$$
x^{\Delta}(t)=A(t) x+F(t, x), x\left(t_{0}\right)=x_{0},
$$

where $x_{0} \in \mathbb{R}^{n}$. Then every solution can be written in the form

$$
x(t)=\Phi_{A}\left(t, t_{0}\right) x\left(t_{0}\right)+\int_{t_{0}}^{t} \Phi_{A}(t, \sigma(s)) F(s, x(s)) \Delta s .
$$

$\mathrm{R}$ e $\mathrm{m}$ a $\mathrm{r} \mathrm{k}$ 3.1. The uniqueness of the solution is ensured since $A(t)$ is a regressive matrix and $f(t)=F(t, x(t))$ is an rd-continuous function for any arbitrary time scale $\mathbb{T}$. For more details, see [6, Theorem 5.8].

Certainly, by using an explicit formula of solutions (26), we are able to study the asymptotic aspect of solutions of nonlinear systems (1), having required some details about trajectory developments of linear systems (2) and growth rate of the perturbed term $F(.,$.$) . That is why, the asymptotic properties of unperturbed$ systems (2) are preserved for perturbed systems (1). We are interested in developing uniform asymptotic stability of the class of nonlinear perturbed time scale systems using appropriate integral inequalities from subsection 3.1. 
First, we consider the class of systems (26) with nonlinear growth disturbance

$$
\|F(t, x)\| \leq \alpha(t)\|x\|+\chi(t)
$$

where $\alpha, \chi(.) \in \mathcal{C}_{r d}\left(\mathbb{T}, \mathbb{R}_{+}\right)$.

Theorem 3.4. Suppose that the linear system (2) is uniformly exponentially stable with constants $\lambda$ and $\gamma$ and the considered functions in (27), $\alpha$ and $\chi$, satisfy the following conditions:

$$
\int_{t_{0}}^{+\infty} \frac{\alpha(s)}{1-\lambda \mu(s)} \Delta s \leq \tilde{d}<+\infty, \int_{t_{0}}^{+\infty} \chi(s) e_{-\lambda}\left(t_{0}, \sigma(s)\right) \Delta s \leq \tilde{k}<+\infty, \quad \forall t_{0} \in \mathbb{T} .
$$

Then the perturbed system is uniformly practically exponentially stable.

P r o o f. Let $t_{0} \in \mathbb{T}, t \in \mathbb{T}_{t_{0}}^{+}$, and $x(t):=x\left(t, t_{0}, x_{0}\right)$ be a solution of system (1) that satisfies the variation of constant formula (26).

Taking into account the nonlinear approximation (27) and the asymptotic aspect trajectories of system (2), we can estimate the solution $x(t)$ :

$$
\|x(t)\| \leq \gamma e_{-\lambda}\left(t, t_{0}\right)\left\|x_{0}\right\|+\gamma e_{-\lambda}\left(t, t_{0}\right) \int_{t_{0}}^{t} e_{-\lambda}\left(t_{0}, \sigma(s)\right)(\alpha(s)\|x(s)\|+\chi(s)) \Delta s .
$$

Applying Lemma 3.2 to the earlier line, we obtain

$$
\begin{gathered}
\|x(t)\| \leq \gamma e_{-\lambda}\left(t, t_{0}\right)\left[\left\|x_{0}\right\|+\gamma\left\{\int_{t_{0}}^{t} e_{-\lambda}\left(t_{0}, \sigma(s)\right)\left(\gamma \alpha(s) e_{-\lambda}\left(s, t_{0}\right)\left\|x_{0}\right\|+\chi(s)\right)\right.\right. \\
\left.\left.\times \exp \left(\gamma \int_{\sigma(s)}^{t} \alpha(r) e_{-\lambda}\left(r, t_{0}\right) e_{-\lambda}\left(t_{0}, \sigma(r)\right) \Delta r\right) \Delta s\right\}\right] .
\end{gathered}
$$

According to the convergence of integrals provided by $\alpha$ and $\chi$, from (28) we extract the estimate

$$
\|x(t)\| \leq \gamma[1+\gamma \tilde{d} \exp [\gamma \tilde{d}]] e_{-\lambda}\left(t, t_{0}\right)\left\|x_{0}\right\|+\gamma \exp [\gamma \tilde{d}] \tilde{k} e_{-\lambda}\left(t, t_{0}\right) .
$$

The above means that the trivial solution of system (1) is uniformly practically exponentially stable with decreasing radius of convergence $r(t)=\gamma \exp [\gamma \tilde{d}] \tilde{k} e_{-\lambda}\left(t, t_{0}\right)$. 
In the following theorem, we present an application of Theorem 3.1 for getting the explicit estimation that emphasizes the required asymptotic behavior for the solutions of the perturbed time scale system (1).

Theorem 3.5. Suppose that the trivial solution of system (2) is uniformly exponentially stable with growth constants $\gamma$ and $\lambda$, the perturbing term $F$ satisfies the growth rate condition

$$
\|F(t, x)\| \leq P(t,\|x\|), t \in \mathbb{T}, \quad x \in \mathbb{R}^{n},
$$

where $P$ verifies $(17)$ and $P(t, 0)=0$ for all $t \in \mathbb{T}$, and there exists a constant $\theta_{0}>0$ such that

$$
\int_{t_{0}}^{+\infty} \frac{Q(s, \theta)}{1-\lambda \mu(s)} \Delta s \leq \tilde{Q}<+\infty, \text { for all }\left(t_{0}, \theta\right) \in \mathbb{T} \times\left[0, \theta_{0}\right] .
$$

Then the trivial solution of the perturbed system (1) is uniformly asymptotically stable.

$\mathrm{P}$ r o o f. Let $t_{0} \in \mathbb{T}$, the solution $x\left(., t_{0}, x_{0}\right)$ verify the integral equation $(26)$,

$$
x(t)=\Phi_{A}\left(t, t_{0}\right) x_{0}+\int_{t_{0}}^{t} \Phi_{A}(t, \sigma(s)) F(s, x(s)) \Delta s, \quad t \in \mathbb{T}_{t_{0}}^{+} .
$$

Then, taking the norms of both sides of (26), we obtain

$$
\|x(t)\| \leq\left\|\Phi_{A}\left(t, t_{0}\right)\right\|\left\|x_{0}\right\|+\int_{t_{0}}^{t}\left\|\Phi_{A}(t, \sigma(s))\right\|\|F(s, x(s))\| \Delta s, \quad t \in \mathbb{T}_{t_{0}}^{+} .
$$

Since the unperturbed system is uniformly exponentially stable with constants $\gamma$ and $\lambda$, the corresponding matrix exponential function satisfies

$$
\left\|\Phi_{A}\left(t, t_{0}\right)\right\| \leq \gamma e_{-\lambda}\left(t, t_{0}\right), \quad t \in \mathbb{T}_{t_{0}}^{+} .
$$

It follows that

$$
\|x(t)\| \leq \gamma e_{-\lambda}\left(t, t_{0}\right)\left\|x_{0}\right\|+\gamma e_{-\lambda}\left(t, t_{0}\right) \int_{t_{0}}^{t} e_{-\lambda}\left(t_{0}, \sigma(s)\right)\|F(s, x(s))\| \Delta s .
$$


Applying Theorem 3.1 and using the upper bound of perturbation (30), we get

$$
\begin{aligned}
\|x(t)\| & \leq \gamma e_{-\lambda}\left(t, t_{0}\right)\left\|x_{0}\right\|+\gamma e_{-\lambda}\left(t, t_{0}\right) \int_{t_{0}}^{t} e_{-\lambda}\left(t_{0}, \sigma(s)\right) P\left(s, \gamma e_{-\lambda}\left(s, t_{0}\right)\left\|x_{0}\right\|\right) \\
& \times \exp \left\{\int_{\sigma(s)}^{t} e_{-\lambda}\left(t_{0}, \sigma(r)\right) Q\left(r, \gamma e_{-\lambda}\left(r, t_{0}\right)\left\|x_{0}\right\|\right) \gamma e_{-\lambda}\left(r, t_{0}\right) \Delta r\right\} \Delta s .
\end{aligned}
$$

Furthermore, from (17), it follows that

$$
e_{-\lambda}\left(t_{0}, \sigma(s)\right) P\left(s, \gamma e_{-\lambda}\left(s, t_{0}\right)\left\|x_{0}\right\|\right) \leq \frac{\gamma Q(s, 0)}{1-\lambda \mu(s)}\left\|x_{0}\right\|, s \in \mathbb{T}_{t_{0}}^{+} .
$$

Combining (32) and (33), we obtain

$$
\begin{aligned}
\|x(t)\| & \leq \gamma e_{-\lambda}\left(t, t_{0}\right)\left\|x_{0}\right\|+\gamma^{2} e_{-\lambda}\left(t, t_{0}\right)\left\|x_{0}\right\|\left\{\int_{t_{0}}^{t} \frac{Q(s, 0)}{1-\lambda \mu(s)}\right. \\
& \left.\times \exp \left\{\gamma \int_{\sigma(s)}^{t} \frac{Q\left(r, \gamma e_{-\lambda}\left(r, t_{0}\right)\left\|x_{0}\right\|\right)}{1-\lambda \mu(r)} \Delta r\right\} \Delta s\right\} .
\end{aligned}
$$

If $\left\|x_{0}\right\|<c:=\frac{\theta_{0}}{\gamma}$, then taking into account (31), we get

$$
\begin{gathered}
\|x(t)\| \leq \gamma e_{-\lambda}\left(t, t_{0}\right)\left\|x_{0}\right\|+e_{-\lambda}\left(t, t_{0}\right) \gamma^{2}\left\|x_{0}\right\| \times \tilde{Q} \exp \{\gamma \tilde{Q}\}, \\
\|x(t)\| \leq\left[\gamma+\gamma^{2} \tilde{Q} \exp (\gamma \tilde{Q})\right] e_{-\lambda}\left(t, t_{0}\right)\left\|x_{0}\right\|, t \in \mathbb{T}_{t_{0}}^{+},\left\|x_{0}\right\|<c,
\end{gathered}
$$

which characterizes the uniform asymptotic stability of the trivial solution of the perturbed system (1) and completes the proof.

Theorem 3.6. Assume that all the statements of Theorem 3.5 are satisfied and condition (31) holds for all $\left(t_{0}, \theta\right) \in \mathbb{T} \times \mathbb{R}_{+}$. Then the trivial solution $x=0$ of the system is globally uniformly asymptotically stable, i.e.,

$$
\left\|x\left(t, t_{0}, x_{0}\right)\right\| \leq \gamma e_{-\lambda}\left(t, t_{0}\right)\left\|x_{0}\right\|, t \in \mathbb{T}_{t_{0}}^{+}, x_{0} \in \mathbb{R}^{n} .
$$

P r o o f. Let $t \in \mathbb{T}_{t_{0}}^{+}$, then the proof follows exactly as in Theorem 3.5 with the observation that

$$
\|x(t)\| \leq \gamma e_{-\lambda}\left(t, t_{0}\right)\left\|x_{0}\right\|+\gamma^{2} e_{-\lambda}\left(t, t_{0}\right)\left\|x_{0}\right\| \int_{t_{0}}^{t} \frac{Q(s, 0)}{1-\lambda \mu(s)}
$$

Journal of Mathematical Physics, Analysis, Geometry, 2015, vol. 11, No. 3 


$$
\times \exp \left\{\gamma \int_{\sigma(s)}^{t} \frac{Q\left(r, \gamma e_{-\lambda}\left(r, t_{0}\right)\left\|x_{0}\right\|\right)}{1-\lambda \mu(r)} \Delta r\right\} \Delta s
$$

Then,

$$
\|x(t)\| \leq \gamma e_{-\lambda}\left(t, t_{0}\right)\left\|x_{0}\right\|+\gamma^{2} \tilde{Q} \exp \{\gamma \tilde{Q}\} e_{-\lambda}\left(t, t_{0}\right)\left\|x_{0}\right\|
$$

Thus,

$$
\left\|x\left(t, t_{0}, x_{0}\right)\right\| \leq\left[\gamma+\gamma^{2} \tilde{Q} \exp (\gamma \tilde{Q})\right] e_{-\lambda}\left(t, t_{0}\right)\left\|x_{0}\right\|, \quad \forall t \in \mathbb{T}_{t_{0}}^{+}, \forall x_{0} \in \mathbb{R}^{n} .
$$

The theorem is proved.

Under rather restrictive conditions, that is, the integral boundedness of upper bound perturbation, using inequalities of solutions given in Lemma 3.4, we obtain the following characterization of the global uniform asymptotic stability.

Corollary 3.7. If the following conditions are satisfied:

(i) equation (2) is uniformly exponentially stable with growth constants $\gamma$ and $\lambda$;

(ii) the perturbed term satisfies the growth bound

$$
\|F(t, x)\| \leq \alpha(t) \beta(\|x\|), t \in \mathbb{T}, x \in \mathbb{R}^{n},
$$

where $\alpha \in \mathcal{C}_{r d}\left(\mathbb{T}, \mathbb{R}_{+}\right), \beta: \mathbb{R}_{+} \rightarrow \mathbb{R}_{+}$a differentiable increasing function with $\beta(0)=0$ and there exists positive constant $\omega_{0}$ such that $\beta^{\prime}$ is decreasing and bounded on $\left[0, \omega_{0}\right]$ with upper bound $M>0$;

(iii)

$$
\int_{t_{0}}^{+\infty} e_{-\lambda}\left(t_{0}, \sigma(s)\right) \alpha(s) \Delta s \leq \tilde{\alpha_{1}}, \int_{t_{0}}^{+\infty} \frac{\alpha(s)}{1-\lambda \mu(s)} \Delta s \leq \tilde{\alpha_{2}}, \quad \forall t_{0} \in \mathbb{T},
$$

then, the trivial solution is uniformly asymptotically stable.

P r o o f. For any initial condition $x_{0}=x\left(t_{0}\right)$, the solution of (1) satisfies

$$
x(t)=\Phi_{A}\left(t, t_{0}\right) x\left(t_{0}\right)+\int_{t_{0}}^{t} \Phi_{A}(t, \sigma(s)) F(s, x(s)) \Delta s .
$$

By the uniform exponential stability of (2) with growth constants $\gamma$ and $\lambda$, the transition matrix verifies $\left\|\Phi_{A}\left(t, t_{0}\right)\right\| \leq \gamma e_{-\lambda}\left(t, t_{0}\right)$ for all $t \in \mathbb{T}_{t_{0}}^{+}$. Taking the norms of both sides and using Lemma 3.4, we have

$$
\|x(t)\| \leq \gamma e_{-\lambda}\left(t, t_{0}\right)\left\|x_{0}\right\|+\gamma e_{-\lambda}\left(t, t_{0}\right) \int_{t_{0}}^{t} \alpha(s) e_{-\lambda}\left(t_{0}, \sigma(s)\right) \beta\left(\gamma e_{-\lambda}\left(s, t_{0}\right)\left\|x_{0}\right\|\right)
$$




$$
\times \exp \left[\int_{\sigma(s)}^{t} \alpha(r) e_{-\lambda}\left(t_{0}, \sigma(r)\right) \beta^{\prime}\left(\gamma e_{-\lambda}\left(r, t_{0}\right)\left\|x_{0}\right\|\right) \gamma e_{-\lambda}\left(r, t_{0}\right) \Delta r\right] \Delta s \text { for all } t \in \mathbb{T}_{t_{0}}^{+} .
$$

It follows that

$$
\begin{aligned}
\|x(t)\| & \leq \gamma e_{-\lambda}\left(t, t_{0}\right)\left\|x_{0}\right\|+\gamma e_{-\lambda}\left(t, t_{0}\right) \int_{t_{0}}^{t} \alpha(s) e_{-\lambda}\left(t_{0}, \sigma(s)\right) \beta\left(\gamma e_{-\lambda}\left(s, t_{0}\right)\left\|x_{0}\right\|\right) \\
& \times \exp \left[\gamma \int_{\sigma(s)}^{t} \frac{\alpha(r)}{1-\lambda \mu(r)} \beta^{\prime}\left(\gamma e_{-\lambda}\left(r, t_{0}\right)\left\|x_{0}\right\|\right) \Delta r\right] \Delta s, \text { for all } t \in \mathbb{T}_{t_{0}}^{+} .
\end{aligned}
$$

Let us consider a constant $\varepsilon>0$.

Since $\beta$ is continuous and $\beta(0)=0$, there exists $\delta_{1}(\varepsilon)>0$ such that

$$
\left\|x_{0}\right\| \leq \min \left\{\frac{\delta_{1}(\varepsilon)}{\gamma}, \frac{\omega_{0}}{\gamma}\right\} \text { implies } \beta\left(\gamma\left\|x_{0}\right\|\right) \exp \left(\gamma \tilde{\alpha}_{2} M\right) \leq \frac{\varepsilon}{2 \gamma \tilde{\alpha}_{1}} .
$$

Denote $\delta(\varepsilon):=\min \left\{\frac{\varepsilon}{2 \gamma}, \frac{\delta_{1}(\varepsilon)}{\gamma}, \frac{\omega_{0}}{\gamma}\right\}$.

If the initial condition satisfies $\left\|x_{0}\right\| \leq \delta(\varepsilon)$, then, $\|x(t)\| \leq \varepsilon \forall t \in \mathbb{T}_{t_{0}}^{+}$.

Consequently, the trivial solution of the perturbed system (1) is uniformly stable.

Moreover, for every initial state with $\left\|x_{0}\right\| \leq c=\frac{\omega_{0}}{\gamma}$, we obtain the estimation

$$
\|x(t)\| \leq \gamma\left[c+\tilde{\alpha}_{1} \beta(\gamma c) \exp \left(\gamma \tilde{\alpha}_{2} M\right)\right] e_{-\lambda}\left(t, t_{0}\right), \forall t \in \mathbb{T}_{t_{0}}^{+} .
$$

So, there exists

$$
T(\varepsilon, c)=-\frac{1}{\lambda} \ln \left(\frac{\varepsilon}{\gamma\left[c+\tilde{\alpha}_{1} \beta(\gamma c) \exp \left(\gamma \tilde{\alpha}_{2} M\right)\right]}\right)
$$

such that for all $t \in\left[t_{0}+T(\varepsilon),+\infty\left[\mathbb{T}\right.\right.$, we get $\left\|x\left(t, t_{0}, x_{0}\right)\right\|<\varepsilon$. It follows that the trivial solution of equation (1) is uniformly asymptotically stable.

To illustrate the useless of integral estimation obtained in Theorem 3.2, we state the previous theorem. Using this integral estimation, we study the stability property pertaining to the case where the initial conditions are arbitrarily enlarged and the solutions of the system converge, in a stable way, to a closed ball centered on the origin, not necessary to the latter. 
Theorem 3.8. If the fundamental matrix of the solution of the linear system of the first approximation satisfies the condition

$$
\left\|\Phi_{A}\left(t, t_{0}\right)\right\| \leq \gamma e_{-\lambda}\left(t, t_{0}\right), \quad t \in \mathbb{T}_{t_{0}}^{+},
$$

with $\gamma \geq 1, \lambda>0,(-\lambda) \in \mathcal{R}^{+}, F$ satisfies the relation

$$
\|F(t, x)\| \leq P(t,\|x\|)+h(t), t \in \mathbb{T}, \quad x \in \mathbb{R}^{n},
$$

$P$ verifies condition $(17), h \in \mathcal{C}_{r d}\left(\mathbb{T}, \mathbb{R}_{+}\right)$is a non-vanishing continuous function satisfying

$$
\int_{t_{0}}^{+\infty} e_{-\lambda}\left(t_{0}, \sigma(s)\right) h(s) \Delta s \leq \tilde{h}<+\infty, \quad \forall t_{0} \in \mathbb{T},
$$

and $Q$ verifies relation (31) for all $\left(t_{0}, \theta\right) \in \mathbb{T} \times \mathbb{R}_{+}$, then the trivial solution is uniformly practically exponentially stable.

P r o o f. By using Theorem 3.2, we can proceed with the same calculation as in the proof of Theorem 3.6 to estimate the norms of $x(t)$. Hence we get

$$
\begin{gathered}
\|x(t)\| \leq \gamma e_{-\lambda}\left(t, t_{0}\right)\left\|x_{0}\right\|+\gamma e_{-\lambda}\left(t, t_{0}\right) \int_{t_{0}}^{t}\left[e_{-\lambda}\left(t_{0}, \sigma(s)\right) P\left(s, \gamma e_{-\lambda}\left(s, t_{0}\right)\left\|x_{0}\right\|\right)\right. \\
\left.+e_{-\lambda}\left(t_{0}, \sigma(s)\right) h(s)\right] \exp \left[\int_{\sigma(s)}^{t} e_{-\lambda}\left(t_{0}, \sigma(r)\right) Q\left(r, \gamma e_{-\lambda}\left(r, t_{0}\right)\left\|x_{0}\right\|\right) \gamma e_{-\lambda}\left(r, t_{0}\right) \Delta r\right] \Delta s \\
\forall t \in \mathbb{T}_{t_{0}}^{+} .
\end{gathered}
$$

Taking into account $(33),(36)$, with a correct choice of $\left\|x_{0}\right\|$, it easy to get $\left\|x\left(t, t_{0}, x_{0}\right)\right\| \leq\left[\gamma+\gamma^{2} \tilde{Q} \exp \{\gamma \tilde{Q}\}\right] e_{-\lambda}\left(t, t_{0}\right)\left\|x_{0}\right\|+\gamma \tilde{h} \exp \{\gamma \tilde{Q}\} e_{-\lambda}\left(t, t_{0}\right), \forall t \in \mathbb{T}_{t_{0}}^{+}$

This estimation implies that the trajectories possess a uniform practical exponential behavior. Thus the theorem is proved.

Motivated by works of S.V. Babenko and A.A. Martynyuk [3] that are interested in the analysis stability of quasi-linear dynamics equations described by (1) with nonlinear perturbation of the form

$$
\|F(t, x)\| \leq a(t)\|x\|^{p} \text { with } a(.) \in \mathcal{C}_{r d}\left(\mathbb{T}, \mathbb{R}_{+}\right)
$$

for $p>1$, we want to cover the situation for $p \in] 0,1[$. We derive a sufficient criteria for the uniform exponential stability using the time scale version of Hölder's inequality. 
Theorem 3.9. If the following conditions are satisfied:

(i) the perturbed term $F(t, x)$ verifies growth condition (37) with $p \in] 0,1[$;

(ii) $\int_{t_{0}}^{\infty}\left\{e_{-\lambda}\left(t_{0}, \sigma(s)\right) a(s)\right\}^{\frac{1}{q}} \Delta s \leq \tilde{a}, \forall t_{0} \in \mathbb{T}$, with $q=1-p$;

(iii) equation (2) is uniformly exponentially stable with positive constants $\lambda$ and $\gamma$, then the trivial solution $x(t) \equiv 0$ of system (1) is uniformly exponentially stable.

$\mathrm{P} \mathrm{r}$ o o f. The solution of the perturbed system is given by a variation of constant formula (26):

$$
x(t)=\Phi_{A}\left(t, t_{0}\right) x\left(t_{0}\right)+\int_{t_{0}}^{t} \Phi_{A}(t, \sigma(s)) F(s, x(s)) \Delta s
$$

or

$$
\|x(t)\| \leq\left\|\Phi_{A}\left(t, t_{0}\right)\right\|\left\|x\left(t_{0}\right)\right\|+\int_{t_{0}}^{t} a(s)\left\|\Phi_{A}(t, \sigma(s))\right\|\|x(s)\|^{p} \Delta s .
$$

We have

$$
\|x(t)\| \leq \gamma e_{-\lambda}\left(t, t_{0}\right)\left\|x\left(t_{0}\right)\right\|+\gamma e_{-\lambda}\left(t, t_{0}\right) \int_{t_{0}}^{t} a(s) e_{-\lambda}\left(t_{0}, \sigma(s)\right)\|x(s)\|^{p} \Delta s
$$

since the homogeneous system is uniformly exponentially stable.

From time scale version of Hölder's inequality [6, Theorem 6.13], we have

$$
\int_{t_{0}}^{t} a(s) e_{-\lambda}\left(t_{0}, \sigma(s)\right)\|x(s)\|^{p} \Delta s \leq\left[\int_{t_{0}}^{t}\left\{a(s) e_{-\lambda}\left(t_{0}, \sigma(s)\right)\right\}^{\frac{1}{q}} \Delta s\right]^{q} \times\left[\int_{t_{0}}^{t}\|x(s)\| \Delta s\right]^{p} .
$$

Then (38) implies

$$
\|x(t)\| \leq \gamma e_{-\lambda}\left(t, t_{0}\right)\left\|x\left(t_{0}\right)\right\|+\gamma e_{-\lambda}\left(t, t_{0}\right)\left[\int_{t_{0}}^{t}\left\{a(s) e_{-\lambda}\left(t_{0}, \sigma(s)\right)\right\}^{\frac{1}{q}} \Delta s\right]^{q} \times\left[\int_{t_{0}}^{t}\|x(s)\| \Delta s\right]^{p} .
$$

By integrating both sides of (39) from $t_{0}$ to $\infty$, we obtain

$$
\int_{t_{0}}^{+\infty}\|x(s)\| \Delta s \leq \gamma \int_{t_{0}}^{+\infty} e_{-\lambda}\left(s, t_{0}\right) \Delta s\left\|x_{0}\right\|+\left[\int_{t_{0}}^{+\infty}\|x(s)\| \Delta s\right]^{p} \times \gamma \int_{t_{0}}^{+\infty} e_{-\lambda}\left(s, t_{0}\right)
$$

Journal of Mathematical Physics, Analysis, Geometry, 2015, vol. 11, No. 3 


$$
\times\left[\int_{t_{0}}^{s}\left\{a(r) e_{-\lambda}\left(t_{0}, \sigma(r)\right)\right\}^{\frac{1}{q}} \Delta r\right]^{q} \Delta s
$$

If

$$
\begin{gathered}
z=\int_{t_{0}}^{+\infty}\|x(s)\| \Delta s, \\
b=\gamma \int_{t_{0}}^{+\infty} e_{-\lambda}\left(s, t_{0}\right) \Delta s\left\|x_{0}\right\|=\frac{\gamma}{\lambda}\left\|x_{0}\right\|
\end{gathered}
$$

and

$$
c=\gamma \int_{t_{0}}^{+\infty} e_{-\lambda}\left(s, t_{0}\right)\left[\int_{t_{0}}^{s}\left\{a(r) e_{-\lambda}\left(t_{0}, \sigma(r)\right)\right\}^{\frac{1}{q}} \Delta r\right]^{q} \Delta s,
$$

then the last inequality shows that the nonnegative number $z$ satisfies the inequality $z \leq b+c z^{p}$. It is easy to see that

$$
z \leq z_{0}
$$

where $z_{0}$ is the unique positive root of the equation $z=b+c z^{p}$.

Moreover, from (40), it follows that

$$
\int_{t_{0}}^{t}\|x(s)\| \Delta s \leq \int_{t_{0}}^{+\infty}\|x(s)\| \Delta s \leq z_{0}
$$

Substituting (41) in inequality (39), we obtain

$$
\|x(t)\| \leq \gamma e_{-\lambda}\left(t, t_{0}\right)\left\|x_{0}\right\|+\gamma z_{0}^{p} \tilde{a}^{q} e_{-\lambda}\left(t, t_{0}\right) \text { for all } t \in \mathbb{T}_{t_{0}}^{+} .
$$

Taking into account that $\mathbb{R}$ is archimedean, we can conclude that there exists $N \in \mathbb{N}$ such that

$$
z_{0}^{p} \leq \frac{N \gamma}{c \lambda}\left\|x_{0}\right\|
$$

Combining (42) and (43), we obtain the desired behavior.

\subsection{Quadratic Stability}

We have studied some types of stability via the time scale version of dynamical integral inequalities. To study the stability of perturbed systems with growth rate conditions of the form (30), we are in position to discuss the asymptotic stability by using direct Lyapunov's method. This approach was developed by 
several authors (see, e.g., [23], [32] [27] and [4]). Motivated by works of Dacunha [13], we give some sufficient conditions for the existence of the quadratic Lyapunov function that ensure the desired asymptotic convergence of trajectories. He was interested in quadratic functions defined for every symmetric matrix $B(t) \in \mathcal{C}_{r d}^{1}\left(\mathbb{T}, \mathbb{R}^{n \times n}\right)$ as $V_{1}(t, x(t))=x^{T}(t) B(t) x(t)$. Obviously, we have the growth rate condition of the perturbed term $F$ in the form

$$
\|F(t, x)\| \leq \alpha(t)\|x(t)\|,
$$

where $\alpha(t):=Q(t, 0)$ for all $t \in \mathbb{T}, x \in \mathbb{R}^{n}$ and $Q$ as defined in relation (17). The asymptotic aspect of any solution $x(t)$ of system (1) results from the evolution of the quadratic energy function mentioned below.

The delta derivative of the quadratic function $V_{1}(t, x(t))$ is given by

$$
\begin{aligned}
{\left[V_{1}(t, x(t))\right]^{\Delta} } & =\left[x^{T}(t) B(t)\right]^{\Delta} x(t)+x^{T}(\sigma(t)) B(\sigma(t)) x^{\Delta}(t) \\
& =x^{T}(t) B^{\Delta}(t) x(t)+x^{T}(t) A^{T}(t) B(t) x(t)+x^{T}(t) B(t) A(t) x(t) \\
& +\mu(t) x^{T}(t) B^{\Delta}(t) A(t) x(t)+\mu(t) x^{T}(t) A^{T}(t) B^{\Delta}(t) x(t) \\
& +\mu(t) x^{T}(t) A^{T}(t) B(t) A(t) x(t)+\mu(t)^{2} x^{T}(t) A^{T}(t) B^{\Delta}(t) A(t) x(t) \\
& +\mu(t) F^{T}(t, x) B(t) F(t, x)+\mu(t)^{2} F^{T}(t, x) B^{\Delta}(t) F(t, x) \\
& +\mu(t) F^{T}(t, x) B(t) A(t) x(t)+\mu(t) x^{T}(t) A^{T}(t) B(t) F(t, x) \\
& +\mu(t) F^{T}(t, x) B^{\Delta}(t) x(t)+\mu(t) x^{T}(t) B^{\Delta}(t) F(t, x) \\
& +F^{T}(t, x) B(t) x(t)+x^{T}(t) B(t) F(t, x) \\
& +\mu(t)^{2} F^{T}(t, x) B^{\Delta}(t) A(t) x(t)+\mu(t)^{2} x^{T}(t) A^{T}(t) B^{\Delta}(t) F(t, x) .
\end{aligned}
$$

Theorem 3.10. If for all $t \in \mathbb{T}$ there exists a positive definite symmetric matrix function $B(t) \in \mathcal{C}_{r d}^{1}\left(\mathbb{T}, \mathbb{R}^{n \times n}\right)$, and $a_{1}, a_{2}, b_{1}, b_{2} \in \mathbb{R}_{+}$such that

(i) $a_{1} I \leq B(t) \leq a_{2} I$,

(ii) $b_{1} I \leq B^{\Delta}(t) \leq b_{2} I$

(iii) $W_{1}(t) \leq 0$,

where

$$
\begin{aligned}
W_{1}(t) & =b_{2} I_{n}+A^{T}(t) B(t)+B(t) A(t)+\mu(t) B^{\Delta}(t) A(t)+B(t) B(t) \\
& +\mu(t) A^{T}(t) B^{\Delta}(t)+\mu(t) A^{T}(t) B(t) A(t)+\mu(t)^{2} A^{T}(t) B^{\Delta}(t) A(t) \\
& +\alpha(t)^{2}\left\{\left(2 \mu(t)^{2}+\mu(t)^{4}+1\right)+\mu(t) a_{2}+\mu(t)^{2} b_{2}\right\} I_{n} \\
& +B^{\Delta}(t) B^{\Delta}(t)+A^{T}(t) B(t) B(t) A(t)+A^{T}(t) B^{\Delta}(t) B^{\Delta}(t) A(t),
\end{aligned}
$$

then system (1) is uniformly stable. 
$\mathrm{P}$ r o o f. $\quad$ Let us consider $x()=.x\left(., t_{0}, x_{0}\right)$, an arbitrary trajectory of system (1).

Define $\Phi: \mathbf{M}_{n, 1}(\mathbb{R}) \times \mathbf{M}_{n}(\mathbb{R}) \times \mathbf{M}_{n, 1}(\mathbb{R}) \rightarrow \mathbb{R}_{+},(x, M, y) \mapsto\left[x^{T} M y+y^{T} M^{T} x\right]$. Using the inequality of polarization, we can estimate certain quantities of the time scale derivative of $\left[V_{1}(t, x(t))\right]^{\Delta}$ :

$$
\begin{gathered}
\Phi\left(x(t), A^{T}(t) B(t), \mu(t) F(t, x)\right) \leq \mu(t)^{2}\|F(t, x)\|^{2}+x^{T}(t) A^{T}(t) B(t) B(t) A(t) x(t), \\
\Phi\left(x(t), B^{\Delta}(t), \mu(t) F(t, x)\right) \leq \mu(t)^{2}\|F(t, x)\|^{2}+x^{T}(t) B^{\Delta}(t) B^{\Delta}(t) x(t), \\
\Phi\left(x(t), A^{T}(t) B^{\Delta}(t), \mu(t)^{2} F(t, x)\right) \leq \mu(t)^{4}\|F(t, x)\|^{2}+x^{T}(t) A^{T}(t) B^{\Delta}(t) B^{\Delta}(t) A(t) x(t), \\
\Phi(x(t), B(t), F(t, x)) \leq\|F(t, x)\|^{2}+x^{T}(t) B(t) B(t) x(t) .
\end{gathered}
$$

Taking the time derivative $\left[V_{1}(t, x(t))\right]^{\Delta}$ and using inequalities (45) and the fact that the perturbed term has the upper bound (44), one obtains

$$
\begin{aligned}
{\left[V_{1}(t, x(t))\right]^{\Delta} } & \leq x^{T}(t)\left\{b_{2} I_{n}+A^{T}(t) B(t)+B(t) A(t)+\mu(t) B^{\Delta}(t) A(t)+B(t) B(t)\right. \\
& +\mu(t) A^{T}(t) B^{\Delta}(t)+\mu(t) A^{T}(t) B(t) A(t)+\mu(t)^{2} A^{T}(t) B^{\Delta}(t) A(t) \\
& +\alpha(t)^{2}\left\{\left(2 \mu(t)^{2}+\mu(t)^{4}+1\right)+\mu(t) a_{2}+\mu(t)^{2} b_{2}\right\} I_{n} \\
& \left.+B^{\Delta}(t) B^{\Delta}(t)+A^{T}(t) B(t) B(t) A(t)+A^{T}(t) B^{\Delta}(t) B^{\Delta}(t) A(t)\right\} x(t) \\
& =x^{T}(t) W(t) x(t) .
\end{aligned}
$$

From (iii), we have

$$
\left[V_{1}(t, x(t))\right]^{\Delta} \leq 0 \text { for all } t \in \mathbb{T}_{t_{0}}^{+} .
$$

By integrating (46) from $t_{0}$ to $t$, one can write

$$
V_{1}(t, x(t)) \leq V_{1}\left(t_{0}, x_{0}\right) \text { for all } t \in \mathbb{T}_{t_{0}}^{+} .
$$

Moreover, we note that from (i) and (47), we obtain

$$
\|x(t)\| \leq \frac{V(t, x(t))}{a_{1}} \leq \sqrt{\frac{a_{2}}{a_{1}}}\left\|x\left(t_{0}\right)\right\| \text { for all } t \in \mathbb{T}_{t_{0}}^{+} .
$$

Consequently, it can be concluded that the perturbed system is uniformly stable. This completes the proof of the theorem.

Theorem 3.11. If for all $t \in \mathbb{T}$ there exists a positive definite symmetric matrix function $B(t) \in \mathcal{C}_{r d}^{1}\left(\mathbb{T}, \mathbb{R}^{n \times n}\right)$, and $a_{1}, a_{2}, b_{1}, b_{2}, c \in \mathbb{R}_{+}$with $\frac{-c}{a_{2}} \in \mathcal{R}^{+}$ such that

(i) $a_{1} I \leq B(t) \leq a_{2} I$,

(ii) $b_{1} I \leq B^{\Delta}(t) \leq b_{2} I$, 
(iii) $W_{1}(t) \leq-c I$,

then system (1) is uniformly exponentially stable.

P r o o f. Consider the Lyapunov function $V_{1}\left(t, x(t)=x^{T}(t) B(t) x(t)\right.$ along an arbitrary trajectory $x\left(t, t_{0}, x_{0}\right)$ of system (1). Following the same approach as in the proof of the previous theorem, we arrive at

$$
\left[V_{1}(t, x(t))\right]^{\Delta} \leq-c\|x(t)\|^{2} \text { for all } t \in \mathbb{T}_{t_{0}}^{+} .
$$

It easy to see that

$$
a_{1}\|x(t)\|^{2} \leq V(t, x) \leq a_{2}\|x(t)\|^{2} \text { for all } t \in \mathbb{T}_{t_{0}}^{+} .
$$

Hence,

$$
\left[V_{1}(t, x(t))\right]^{\Delta} \leq-\frac{c}{a_{2}} V(t, x(t)) \text { for all } t \in \mathbb{T}_{t_{0}}^{+} .
$$

Using Comparison Theorem 2.1, we obtain the following estimation from (48):

$$
V_{1}(t, x(t)) \leq V\left(t_{0}, x_{0}\right) e_{-\frac{c}{a_{2}}}\left(t, t_{0}\right) \quad \text { for all } t \in \mathbb{T}_{t_{0}}^{+} .
$$

Consequently,

$$
\|x(t)\| \leq \sqrt{\frac{a_{2}}{a_{1}}}\left\|x\left(t_{0}\right)\right\| \sqrt{e_{-\frac{c}{a_{2}}}\left(t, t_{0}\right)} \quad \text { for all } t \in \mathbb{T}_{t_{0}}^{+} .
$$

Immediately, the trivial solution of the perturbed system is uniformly exponentially stable with rate $-\frac{c}{a_{2}}$. This finishes the proof of the theorem.

According to the control analysis, the stability of dynamical control systems is the main feature that should be taken to the consideration while choosing the design of the system. By using a closed-loop design, we can study the exponential stabilization with a linear state feedback control $u(t)=K(t) x(t)$ of nonlinear systems of the form

$$
x^{\Delta}(t)=A(t) x(t)+C(t) u(t)+f(t, x(t), u(t)),
$$

where $K: \mathbb{T} \rightarrow \mathbb{R}^{n \times n}, C: \mathbb{T} \rightarrow \mathbb{R}^{n \times m}$ are variant matrices $(m \leq n)$ and $f$ is an rd-continuous function.

Take into account that the perturbed term $f$ satisfies a linear growth condition similar to (44),

$$
\|f(t, x, u)\| \leq \zeta(t)\|x(t)\|,
$$

where $\zeta \in \mathcal{C}_{r d}\left(\mathbb{T}, \mathbb{R}_{+}\right), x \in \mathbb{R}^{n}$ and $u \in \mathbb{R}^{m}$. For the consideration of the existence and uniqueness of solutions of the admitted class of systems, as proved in $[6$, 
section 8.2], we suppose that the matrix function $A+C K$ is regressive, $f$ being rd-continuous. Related to Theorem 3.11, for the explicit form of $W_{1}(t)$, we replace $A(t)$ by $A(t)+C(t) K(t)$ to get a new expression denoted by $W_{2}(t)$. In this situation, using the closed loop design, we can postulate this result for (50).

Proposition 3.1. If for all $t \in \mathbb{T}$ there exists a positive definite symmetric matrix function $B(t) \in \mathcal{C}_{r d}^{1}\left(\mathbb{T}, \mathbb{R}^{n \times n}\right)$, and $a_{1}, a_{2}, b_{1}, b_{2}, c \in \mathbb{R}_{+}$with $\frac{-c}{a_{2}} \in \mathcal{R}^{+}$ such that

(i) $a_{1} I \leq B(t) \leq a_{2} I$,

(ii) $b_{1} I \leq B^{\Delta}(t) \leq b_{2} I$,

(iii) $W_{2}(t) \leq-c I$

then the nonlinear control system (50) is uniformly exponentially stabilizable by the control $u(t)=K(t) x(t)$.

P r o o f. We consider system (50) in a closed loop with the feedback controller $u(t)=K(t) x(t)$, we obtain a system as in (1), and then, by using Theorem (3.11), we can deduce that $u(t)=K(t) x(t)$ stabilizes system (50) uniformly and exponentially .

Now we consider the Lyapunov function for system (1):

$$
V_{3}(t, x(t))=\langle B(t) x(t), x(t)\rangle+\|x(t)\|^{2} .
$$

The delta derivative of the scalar function

$$
V_{2}(t, x(t))=\|x(t)\|^{2}=x^{T}(t) x(t)
$$

with respect to $t$ has the form

$$
\begin{aligned}
{\left[V_{2}(t, x(t))\right]^{\Delta} } & =\left[x^{T}(t)\right]^{\Delta} x(t)+x^{T}(\sigma(t)) x^{\Delta}(t) \\
& =x^{T}(t)\left[A^{T}(t)+A(t)+\mu(t) A^{T}(t) A(t)\right] x(t) \\
& +F^{T}(t, x) x(t)+x^{T}(t) F(t, x) \\
& +\mu(t) x^{T}(t) A^{T}(t) F(t, x)+\mu(t) F^{T}(t, x) A(t) x(t) \\
& +\mu(t)\|F(t, x)\|^{2} .
\end{aligned}
$$

Theorem 3.12. If there exists a positive definite symmetric matrix function $B(t) \in \mathcal{C}_{r d}^{1}\left(\mathbb{T}, \mathbb{R}^{n \times n}\right)$, and $a_{1}, a_{2}, b_{1}, b_{2}, c \in \mathbb{R}_{+}$such that

(i) $a_{1} I \leq B(t) \leq a_{2} I$, 
(ii) $b_{1} I \leq B^{\Delta}(t) \leq b_{2} I$

(iii) $W_{3}(t) \leq 0$,

where

$W_{3}(t)=A^{T}(t)+A(t)+(1+\mu(t)) A^{T}(t) A(t)+\left\{1+\alpha(t)^{2}\left(1+\mu(t)+\mu(t)^{2}\right)\right\} I_{n}+W_{1}(t)$,

then system (1) is uniformly stable.

$\mathrm{P}$ r o o f. Let us consider $x(t):=x\left(t, t_{0}, x_{0}\right)$, an arbitrary solution of system (1). Now we are in a position to show that $V(t, x(t))$, as defined in (52), is a Lyapunov function.

To verify this, we substitute the above inequalities with growth rate condition of the perturbed term and obtain

$$
\begin{aligned}
F^{T}(t, x) x(t)+x^{T}(t) F(t, x) & \leq\|x(t)\|^{2}+\|F(t, x)\|^{2} \\
\Phi\left(x(t), A^{T}(t), \mu(t) F(t, x)\right) & \leq \mu(t)^{2}\|F(t, x)\|^{2}+x^{T}(t) A^{T}(t) A(t) x(t) .
\end{aligned}
$$

The delta derivative of the Lyapunov function $V_{3}$ along the trajectories of system (1) is given by

$$
\left[V_{3}(t, x(t))\right]^{\Delta}=\left[V_{1}(t, x(t))\right]^{\Delta}+\left[V_{2}(t, x(t))\right]^{\Delta} .
$$

Taking into consideration relations (45), (53), $(i)$ and $(i i)$, we get

$$
\left[V_{3}(t, x(t))\right]^{\Delta} \leq x^{T}(t) W_{3}(t) x(t) \leq 0 .
$$

Analogously as in the proof of Theorem 3.10, the required feature of the stability holds. The proof is completed.

Theorem 3.13. If there exists a positive definite symmetric matrix function $B(t) \in \mathcal{C}_{r d}^{1}\left(\mathbb{T}, \mathbb{R}^{n \times n}\right)$, and $a_{1}, a_{2}, b_{1}, b_{2}, c \in \mathbb{R}^{+}$with $\frac{-c}{a_{2}} \in \mathcal{R}^{+}$such that

(i) $a_{1} I \leq B(t) \leq a_{2} I$,

(ii) $b_{1} I \leq B^{\Delta}(t) \leq b_{2} I$,

(iii) $W_{3}(t) \leq-c I$,

then system (1) is uniformly exponentially stable.

P r o o f. The proof follows exactly as in Theorem 3.11, with the observation that the Lyapunov function $V_{3}$ decreases exponentially over time in evolution. We omit the details. 
Of course, the problem at hand is obtaining a proper scalar function. Now we can introduce a new form of the energy scalar function below.

Definition 3.1. Let $B(t)$ be a symmetric matrix such that $B(t) \in \mathcal{C}_{r d}^{1}\left(\mathbb{T}, \mathbb{R}^{n \times n}\right)$ and $V_{4}$ be a candidate energy function given by

$$
V_{4}(t, x(t))=x^{T}(t) B(t) x(t)+a(\|x(t)\|),
$$

where $a: \mathbb{R}_{+} \rightarrow \mathbb{R}_{+}$a differentiable strictly increasing function with $a(0)=0$.

The delta derivative of $V$ along the trajectory of system (1) is given by

$$
\left[V_{4}(t, x(t))\right]^{\Delta}=\left[x^{T} B(t) x(t)\right]^{\Delta_{t}}+[a(\|x(t)\|)]^{\Delta_{t}} .
$$

So, there exists $c$ in the real interval $[t, \sigma(t)]$ such that

$$
[a(\|x(t)\|)]^{\Delta_{t}}=\frac{a^{\prime}(\|x(c)\|)}{2\|x(c)\|}\left[x^{T}(t) x(t)\right]^{\Delta_{t}} .
$$

We define the operator $\omega(t):=\frac{a^{\prime}(\|x(c)\|)}{2\|x(c)\|}$ for $t \in \mathbb{T}$.

Theorem 3.14. If there exists a positive definite symmetric matrix function $B(t) \in \mathcal{C}_{r d}^{1}\left(\mathbb{T}, \mathbb{R}^{n \times n}\right)$, and $a_{1}, a_{2}, b_{1}, b_{2} \in \mathbb{R}_{+}$such that

(i) $a_{1} I \leq B(t) \leq a_{2} I$,

(ii) $b_{1} I \leq B^{\Delta}(t) \leq b_{2} I$,

(iii) $W_{4}(t) \leq 0$,

where

$W_{4}(t)=\omega(t)\left[A^{T}(t)+A(t)+(1+\mu(t)) A^{T}(t) A(t)+\left\{1+\alpha(t)^{2}\left(1+\mu(t)+\mu(t)^{2}\right)\right\} I_{n}\right]+W_{1}(t)$, then system (1) is uniformly stable.

$\mathrm{P} \mathrm{r}$ o o $\mathrm{f}$. Let us consider $x(t):=x\left(t, t_{0}, x_{0}\right)$, an arbitrary solution of system (1). Now it is possible to show that $V_{4}(t, x(t))$, as defined in (55), is a decreasing function. Using the same approach of the proof as in the precedent theorem, we arrive at

$$
V_{4}^{\Delta}(t, x(t)) \leq 0 \text { for all } t \in \mathbb{T}_{t_{0}}^{+} .
$$

Integrating it from $t_{0}$ to $t$, we get $V(t, x(t)) \leq V\left(t_{0}, x_{0}\right)$. Combine the inequality above with relation (i),

$$
V_{4}(t, x(t)) \leq a_{2}\left\|x_{0}\right\|^{2}+a\left(\left\|x_{0}\right\|\right):=\beta\left(\left\|x_{0}\right\|\right) .
$$


It is trivial to see that $\beta($.$) is a strictly increasing continuous function. Conse-$ quently,

$$
\|x(t)\| \leq \frac{1}{a_{1}} \sqrt{\beta\left(\left\|x_{0}\right\|\right)} \text { for all } t \in \mathbb{T}_{t_{0}}^{+},
$$

which implies that the trivial solution of system (1) is uniformly stable.

\section{Conclusion}

The intent of this paper is to develop some growth rate conditions on the nonlinear perturbations, which provide a uniform asymptotic convergence of trajectories, using the appropriate integral inequalities on time scales. Moreover, we use the linear matrix inequality approach to infer the asymptotic stability for perturbed time scale systems.

\section{References}

[1] V.M. Alekseev, An Estimate for the Perturbations of Solutions of Ordinary Differential Equations. - Vestnik Moskov. Univ. Ser. I. Mat. Meh. (1961), No. 2, 28-36. (Russian)

[2] B. Aulbach and S. Hilger, Linear Dynamic Processes with Inhomogeneous Time Scale, Nonlinear Dynamics and Quantum Dynamical Systems. (Gaussing, 1990), Math. Res. Vol. 59, Akademie Verlag, Berlin, 1990, p. 9-20.

[3] S.V. Babenko and A.A. Martynyuk, Nonlinear Dynamic Inequalities and Stability of Quasilinear Systems on Time Scales. - Nonlinear Dynamics and Systems Theory 13 (2013), No. 1, 13-23.

[4] S.V. Babenko, Revisiting the Theory of Stability on Time Scales of the Class of Linear Systems with Structural perturbations. - Internationnal Applied Mechanics 47 (2011), No. 1, 86-96.

[5] A. Ben Abdallah, M. Dlala, and M.A. Hammami, A New Lyapunov Function for Stability of Time-Varying Nonlinear Perturbed Systems. - Syst. Control Lett. 56 (2007), No. 3, 179-187.

[6] M. Bohner and A. Peterson, Dynamic Equations on Time Scales: An Introduction with Applications. Birkhäuser Boston, 2001.

[7] M. Bohner and A. Peterson, Advances in Dynamic Equations on Time Scales. Birkhäuser Boston, 2003.

[8] M. Bohner and A.A. Martynyuk, Elements of Stability Theory of A.M. Liapunov for Dynamic Equations on Time Scales. - Nonlinear Dynamics and Systems Theory 7 (2007), No. 3, 225-251.

[9] M. Bohner, Some Oscillation Criteria for First Order Delay Dynamic Equations. Far East J. Appl. Math. 18 (2005), No. 3, 289-304. 
[10] F. Brauer and J.A. Nohel, The Qualitative Theory of Ordinary Differential Equations. An Introduction. New York, NY, USA, Dover Publications, 1989.

[11] S.K. Choi, D.M. Im, and N. Koo, Stability of Linear Dynamic Systems on Time Scales. Advances in Difference Equations. Article ID 670203, 2008.

[12] J.J. DaCunha, Transition Matrix and Generalized Matrix Exponential Via the Peano-Baker Series. - J. Difference Equ. Appl. 11 (2005), No. 15, 1245-1264.

[13] J.J. DaCunha, Stability for Time Varying Linear Dynamic Systems on Time Scales. — J. Comput. Appl. Math. 176 (2005), No. 2, 381-410.

[14] M. Dlala and M.A. Hammami, Uniform Exponential Practical Stability of Impulsive Perturbed Systems. - J. Dynamical and Control Systems 13 (2007), No. 3, 373-386.

[15] B. Ghanmi, N. Hadj Taieb, and M.A. Hammami, Growth Conditions for Exponential Stability of Time-Varying Perturbed Systems. - Int. J. Control 86 (2013), No. 6, 1086-1097.

[16] S. Hilger, Ein Maßkettenkalkül mit Anwendung auf Zentrumsmannigfaltigkeiten. Ph. D. thesis, Universität Würzburg, 1988.

[17] S. Hilger, Analysis on Mesure Chains $-a$ Unified Approach to Continuous and Discrete Calculus. - Results Math. 18 (1990), No. 1/2, 18-56.

[18] S. Hilger and P.E. Kloeden, Comparative Time Grainyness and Asymptotic Stability of Dynamical Systems. - Autom. Remote Control 55 (1994), No. 1, 1293-1298.

[19] J. Hoffacker and C.C. Tisdell, Stability and Instability for Dynamic Equations on Time Scales. - Comput. Math. Appl. 49 (2005), No. 9/10, 1327-1334.

[20] B. Kaymakcalan, Lyapunov Stability Theory for Dynamic Systems on Time Scales. - J. Appl. Math. Stochastic Anal. 5 (1992), No. 3, 275-281.

[21] V. Lakshmikantham, S. Sivasundram, and B. Kaymakcalan, Dynamic Systems on Measure Chains. Kluwer Academic Publishers, Boston, Mass, USA, 1996.

[22] V. Lakshmikantham and S. Leela, Differential and Integral Inequalities. Vol. 1. New York, NY, USA, Academic Press, 1969.

[23] Lei Li and Shi Huang Hong, Exponential Stability for Set Dynamic Equations on Time Scales. - J. Comput. Appl. Math. 235 (2011), No. 17, 4916-4924.

[24] W.N. Li, Some Pachpatte Type Inequalities on Time Scales. - Comput. Math. Appl. 57 (2009), No. 2, 275-282.

[25] Li Yin and Feng Qi, Some Integral Inequalities on Time Scales. - Results Math. 64 (2013), No. 3/4, 371-381.

[26] M.E. Lord and A.R. Mitchell, A New Approach to the Method of Nonlinear Variation of Parameters. - J. Appl. Math. Com. 4 (1978), No. 2, 95-105.

[27] A.A. Martynyuk, On the Exponential Stability of a Dynamical System on a Time Scale. - Dokl. Akad. Nauk 421 (2008), No. 3, 312-317. (Russian) 
[28] Yu.A. Martynyuk-Chernienko and L.N. Chernetskaya, Analyzing the Exponential Stability of Motion on Time Scales. - Int. Appl. Mech. 46 (2010), No. 4, 467-473.

[29] B.G. Pachpatte, Inequalities for Differential and Integral Equations. Mathematics in Science and Engineering. 197, Academic Press Inc. San Diego CA, 1998.

[30] D.B. Pachpatte, Explicit Estimates on Integral Inequalities with Time Scale. J. Inequal. Pure Appl. Math. 7 (2006), No. 4, Article 143.

[31] A. Peterson and R.F. Raffoul, Exponential Stability of Dynamic Equations on Time Scales. - Adv. Differ. Equ. Appl. 2 (2005), 133-144.

[32] Yjun Ma and Jitao Sun, Uniform Eventual Lipshitz Stability of Impulsive Systems on Time Scales. - J. Comput. Appl. Math. 211 (2009), No. 1, 246-250. 